\title{
A word from the Editors on Protocol Review
}

\author{
The lab animal community lost Dr. Jerald Silverman on November 14, 2020 to COVID-19. Here, we reflect on Jerry \\ and his long-running and well-loved Protocol Review column.
}

F or those of you who conduct or oversee animal research in the United States, there are a number of laws, regulations, guidelines, and best practices to navigate, each in place to ensure the welfare of your animals is given its due consideration. Ample resources exist to help you decide how you should approach a scenario as your facility's protocols are designed and approved and an animal study gets underway - there are guidebooks, FAQs, webinars, and meetings - but when it comes down to it, you may find that your IACUC might interpret a guideline a little differently than another, that the 'answer' is not entirely clear cut. What is the right thing to do, for your animals and your research program?

For three decades, Jerald Silverman has posed hypothetical scenarios devised to probe the grey areas of US animal research rules and regulations in his monthly Protocol Review column. The editors of Lab Animal - both past and present are sad to mark the end of an era with Jerry's passing from COVID-19 on November 14, 2020.

Over those years, many of you may have received an email from Jerry, inquiring if you'd like to participate in the column. Attached will have been a short prompt, describing a situation involving some aspect of animal research and frequently occurring at the fictitious Great Eastern University. Is that analgesic appropriate? What's the pain category here? Does that misconduct or unexpected issue need to be reported, and to whom? At the end of each scenario, Jerry asked: how would you respond?
Though hypothetical, the scenarios were frequently grounded in truth - over his long career as a lab animal veterinarian and co-editor of three editions of the IACUC Handbook, Jerry had encountered more than his fair share of quirky protocol proposals and unexpected kinks in the course of a research study.

Respondents were encouraged to cite the resources they rely on and explain how they would react to the proposed situation; as applicable, representatives of the appropriate oversight bodies - the National Institutes of Health Office of Laboratory Animal Welfare (NIH OLAW), the USDA's Animal and Plant Health Inspection Service (USDA APHIS), and occasionally AAALAC International would weigh in too, offering their guidance and gently correcting any misinterpretations.

At the annual AALAS meetings, Protocol Review always came up as an invaluable resource, eagerly awaited and shared among staff once the latest issue finally arrived. Jerry would turn up at AALAS too - an incredibly kind, funny man but quick to the point. It was a pleasure when he popped by the booth, and when his column appeared in our inboxes each month.

Over all those years writing the Protocol Review prompts, he touched on it all. The animals in question have included mice and rats and monkeys; cats and dogs, frogs and fish; livestock - pigs and sheep and llamas; even wildlife. He asked what you thought about the scientific justification for a proposed protocol, how you determine the optimal number of animals needed for a project, and what to do with (inevitable?) deviations from the plan. He posed problems concerning quorums and ad hoc approvals, and how you would manage financial disputes and interpersonal relationships. In 2004 he even noted the rise of virtual interactions - long before we were all relegated to Zoom meetings.

Our digital archive only extends back to 2003, but for those of you holding on to your print copies of Lab Animal, you'll find Jerry's name stretching back to the late 80 s.

Recently, Jerry had shared that he was, at long last, starting to run out of ideas, and we knew Protocol Review, at least in its current format, was likely nearing its end - how we wish it had gone on Jerry's own terms. Jerry's final Protocol Review column, which ran last month, covered that topic that's been on our minds for nearly a year now and is made that much more tragic by the circumstances.

We are left with a hole in our pages that we aren't yet sure how we will fill, but we want to thank all of our readers who have participated in Protocol Review over the years, and we hope you took the time to think about how your own facility would respond to the topic of the month - you just never know what might come up.

Most importantly, we remember Jerry for his diligence, his dedication to the lab animal field, and his tireless efforts to help others navigate the twists and turns of their own protocol reviews. He will be dearly missed.

Published online: 12 January 2021 https://doi.org/10.1038/s41684-021-00708-z 